\title{
The Comparison of the Effect of Oat and Shiitake Mushroom Powder to Prevent Body Weight Gain in Rats Fed High Fat Diet
}

\author{
Dian Handayani ${ }^{1,2}$, Barbara J. Meyer ${ }^{1}$, Jiezhong Chen ${ }^{1}$, Patricia Tang ${ }^{3}$, Philip Chi Lip Kwok ${ }^{4}$, \\ Hak-Kim Chan ${ }^{3}$, Xu-Feng Huang ${ }^{1^{*}}$ \\ ${ }^{1}$ Metabolic Research Centre, School of Health Sciences and Illawarra Health and Medical Research Institute (IHMRI), University of \\ Wollongong, Wollongong, Australia; ${ }^{2}$ Nutrition Departments, Faculty of Medicine, University of Brawijaya, Malang, Indonesia; \\ ${ }^{3}$ Faculty of Pharmacy, The University of Sydney, Sydney, Australia; ${ }^{4}$ Department of Pharmacology and Pharmacy, Li Ka Shing Fac- \\ ulty of Medicine, The University of Hong Kong, Hong Kong, China. \\ Email: *xhuang@uow.edu.au
}

Received May 25 ${ }^{\text {th }}$, 2012; revised June 25 ${ }^{\text {th }}$, 2012; accepted July $3^{\text {rd }}, 2012$

\begin{abstract}
Preventing obesity could be done by lowering plasma TAG that inhibits adipogenesis. Oat and mushroom beta-glucans in the diet has been reported to lower plasma lipid; however the data focusing on their effects on TAG and obesity are insufficient. In the present study, lowering plasma triacylglycerol, fat deposition, body weight gain (BWG) in rats fed a high fat diet (HFD) was evaluated. Rats in the control group were given HFD only and rats in the treatment group fed HFD enriched with $0.2 \%, 0.6 \%$ and $1.8 \%$ (wt:wt) beta-glucan from oats (LD-O, MD-O, HD-O) or mushroom (LD-M, MD-M, HD-M). After 6 weeks dietary intervention, the rats fed HD-M showed significantly lower plasma TAG, total fat mass, white adipose tissue, inguinal fat and BWG level more than HD-O treated rats $(p<0.05)$. The underlying mechanism in lowering plasma TAG, fat pad masses and BWG in HD-M was increasing ratio of fat faecal to faecal weight which was significantly higher than HD-O $(p<0.05)$. This study demonstrated that the preventing obesity via lowering plasma TAG and fat deposition was different depending on beta-glucan origin, either from oats and Shiitake mushroom.
\end{abstract}

Keywords: Beta-Glucan; Triacylglycerol; Fat Deposition; Obesity; Dietary Fibre

\section{Introduction}

In recent years, the prevalence of obesity has been widely reported to induce metabolic syndrome, cardiovascular disease, diabetes mellitus and cancer [1-3]. It has been reported that lowering plasma triacylglycerol (TAG) levels will be efficient in combating body weight gain as an increased plasma TAG level was significantly correlated with increase in body mass index and fat pad masses on rats [4]. Beta-glucan is well documented as lowering plasma lipids in human [5-8] and animal $[9,10]$ studies.

Oats and mushrooms have been found to be good sources of beta-glucan. An enrichment diet of oats in a clinical study was not reported to lower plasma TAG levels [11-14] but lowered total plasma cholesterol (TC) [11,13]. Limited human studies such as [15] have shown the prevention of increased TAG plasma levels using a mushroom enriched diet.

One of the mechanisms of oat beta-glucan in lowering

*Corresponding author. plasma lipid is its capacity to increase food viscosity [9, 10,16]. By increasing viscosity of food in the lumen, beta-glucan was reported to prevent and delay the absorption of nutrients. However, the viscosity of beta-glucan is dependent on the molecular weight, molecular structure, and food matrix $[7,10,17]$.

While in nearly all of the reported research beta-glucan was found to be the main component from oats with the potential to lower plasma lipid, in contrast, in research using the shiitake mushroom, not only was beta-glucan reported to lower lipid, but other nutrient content such as eritadenine also lowered plasma lipids [18-20]. Another difference between oats and shiitake mushrooms is the molecular structure of their beta-glucan [21].

Thus it will be predicted from compositional data that, when comparable amounts of beta-glucan are consumed, shiitake mushrooms will lower plasma lipid more effectively than oats. These predictions are supported by the findings of an extraction of each component of shiitake mushrooms; for instance, polysaccharide, and eritadenine 
lower plasma TAG by 15\% [22], and 39\% [23] respectively. Also reported exoskeleton mushroom contains chitin-chitosan lower plasma TAG lipid by 32\% [24].

To the best of our knowledge, no data are available to examine the comparative effect of oats and shiitake mushrooms in preventing body weight gain in a similar designed study. Such a study would use equivalent dosages of beta-glucan for both the oat and mushroom powder, and in addition, the same background high fat diet (HFD) would be given and the same type of food matrix used to administer the oats and mushrooms.

The aims of this research were to 1) compare the effect of oats and mushrooms powder to prevent body weight gain, 2) to determine the effective dose of oat and mushroom powder necessary to prevent body weight gain and 3 ) to determine the potential underlying mechanisms to preventing body weight gain.

\section{Material and Methods}

\subsection{Animals and Diet}

Seventy male Wistar rats at 9 weeks of age were purchased from the Animal Resource Centre (ARC)-Perth, Western Australia. All experimental procedures were approved by the Animal Ethics Committee of the University of Wollongong, AE 09/01. The animals were housed two rats per cage in environmentally controlled conditions (temperature $22^{\circ} \mathrm{C}$, light cycle from 06:00 to 18:00 hours and dark cycle from 18:00 to 06:00 hours) and had ad libitum access to food and water. Rats were fed a commercial chow (rat and mouse YS Feed, Young, NSW) in order to acclimatise to the new facility and environment. The seventy rats were divided into seven groups $(\mathrm{n}=10)$ and fed $\sim 50 \%$ HFD with additional nil, low, medium and high doses of oat powder and Shiitake mushroom powder 1) HFD, 2) low dose oat (LD-O), 3) medium dose oat (MD-O) and 4) high dose oat (HD-O), 5) low dose Shiitake mushroom (LD-M), 6) medium dose Shiitake mushroom (MD-M), 7) high dose Shiitake mushroom (HD-M). Oat bran powder was from OatWell ${ }^{\circledR}$ Crea-nutrition and the Shiitake mushroom was from Shenzen Dashan, Food Stuff. Co. Ltd., Shanghai that was imported by a local store in Australia. The nutrient composition of the oats and mushrooms is shown in Table 1. The low, medium and high doses of either oats or mushrooms contain beta-glucan that is comparable to 3,9 and $27 \mathrm{~g}$ of the beta-glucan requirement for humans, respectively. The composition of the seven diets is shown in Table 2.

\subsection{Body Weight, Food Intake and Fat Pad Masses}

Animals were weighed weekly throughout the six week intervention period. Food intake was measured every 24 hours by weighing the amount of total food (g) provided to the rats and subtracting the remaining food (g) in the cage after 24 hours.

After completion of food intervention, rats were sacrificed via carbon dioxide asphyxiation. The white adipose tissue (WAT) comprised of visceral fat (epididymal, perirenal, and omental fat) and subcutaneous fat (inguinal fat) were then removed and weighed.

\subsection{Plasma Lipid Profile}

Blood samples were obtained by puncturing the right ventricle of the heart and collected in EDTA coated tubes and centrifuged at $3000 \mathrm{rpm}$ at $22^{\circ} \mathrm{C}$ for 25 minute. High density lipoprotein (HDL) was isolated from plasma with dextran sulphate and magnesium chloride, based on a modified method from Sjoblom and Eklund [25]. Total plasma cholesterol (TC), TAG, high density lipoprotein (HDL) and plasma Non Esterified Fatty acid (NEFA) were measured using the Konelab ${ }^{\circledR}$ 20XT automatic analyser. The TC, TAG and HDL were analysed with Infinity $^{\mathrm{TM}}$ reagent from Thermo Fisher Scientific (Auburn NSW, Australia) and plasma NEFA were analysed use a highly specific enzymatic (Wako Chemical, Richmond, VA, USA).

\subsection{Viscosity and Molecular Weight (MW) from Oat and Mushroom Beta-Glucan}

\subsubsection{Extraction of Beta-Glucan}

Beta-glucans were extracted from commercial varieties of oats and shiitake mushrooms. Oats and Shiitake mushrooms were pulverized separately using a blender (Breville, Sydney, Australia) and sieved through $355 \mu \mathrm{m}$ mesh. Different concentrations of oats and mushrooms (Table 3) were prepared by stirring a measured amount of each powder in de-ionised water at $70^{\circ} \mathrm{C}$ for $30 \mathrm{~min}$ utes. The suspensions were then centrifuged in a Sorvall RC-5B centrifuge (DuPont Company, Wilmington, Delaware, USA) at 10,000 rpm for 30 minutes. The clear viscous supernatant solutions, containing extracted beta-glucans, were collected for viscosity measurement.

\subsubsection{Viscosity Measurement}

The apparent viscosity of the extract was determined at a constant temperature of $37^{\circ} \mathrm{C}$, to mimic the human body temperature, using a DV III Rheometer (Brookfield, Middleboro, Massachusetts, USA). A water-jacketed sample cup was employed to maintain a constant sample temperature during the measurement. The speed of the rotating spindle was increased and then decreased to the starting speed. The speeds were chosen so that the torque exerted on the liquid was between the manufacturer's 

in Rats Fed High Fat Diet

Table 1. Nutrient information of OatWell ${ }^{\circledR}$ and Shiitake mushroom.

\begin{tabular}{|c|c|c|c|}
\hline Nutritive Value & & OatWell $^{\mathrm{a}}$ & Shiitake Mushroom \\
\hline \multirow[t]{6}{*}{ Chemical Properties } & Protein (g) & 20 & $20^{\mathrm{b}}$ \\
\hline & Carbohydrate (g) & 22 & $32.2^{\mathrm{b}}$ \\
\hline & Total dietary fiber (g) & 44 & $33.3^{\mathrm{b}}$ \\
\hline & Beta-glucan soluble fibre (g) & 22 & $30^{c}$ \\
\hline & Fat (g) & 5 & $0.7^{\mathrm{b}}$ \\
\hline & Energy Kcal or Kj & 215/899 & $282 / 1178^{\mathrm{b}}$ \\
\hline \multirow[t]{15}{*}{ Vitamins and Minerals } & Sodium (Na) (mg) & 8 & $30^{\mathrm{d}}$ \\
\hline & Magnesium (Mg) (mg) & 250 & $1550[26]$ \\
\hline & Calcium (Ca) (mg) & 120 & $500[26]$ \\
\hline & Potassium (K) (mg) & 680 & $26.7[26]$ \\
\hline & Iron (Fe) (mg) & 11 & $33[26]$ \\
\hline & Zinc (Zn) (mg) & 6 & $92[26]$ \\
\hline & Vitamin A ( $\mu \mathrm{g})$ & NA & NA \\
\hline & Vitamin B1(mg) & 0.3 & $0.6[26]$ \\
\hline & Vitamin B2 (mg) & NA & $1.8[26]$ \\
\hline & Vitamin B6 (mg) & 0.9 & NA \\
\hline & Vitamin B12 ( $\mu \mathrm{g})$ & NA & $0.8[26]$ \\
\hline & Folic Acid $(\mu \mathrm{g})$ & 44 & $640[26]$ \\
\hline & Vitamin C (mg) & NA & $25[26]$ \\
\hline & Vitamin D ( $\mu \mathrm{g})$ & NA & $1 \mu \mathrm{g}[26]$ \\
\hline & Vitamin E (delta-alpha-tocopherol) & $0.1 \mathrm{mg}$ & NA \\
\hline \multirow[t]{3}{*}{ Fatty Acids } & Saturated FA (g) & 0.9 & $0.3^{\mathrm{d}}$ \\
\hline & Polyunsaturated FA (g) & 2.0 & NA \\
\hline & Monounsaturated FA (g) & 2.1 & NA \\
\hline \multicolumn{4}{|l|}{ Other Specification } \\
\hline Molecular Weight & $\mathrm{DA}=$ Dalton $=\mathrm{g} / \mathrm{Mol}$ & $1689.000[27]$ & $>1689.000$ \\
\hline \multirow[t]{3}{*}{ Other Active Components } & Chitin (\%) & - & $5.36[28]$ \\
\hline & Eritadenine (\%) & - & $0.3-0.63[29]$ \\
\hline & Chitosan & - & - \\
\hline
\end{tabular}

Data as per $100 \mathrm{~g}$ on dry basis. ${ }^{\mathrm{a}}$ Information based on product data sheet OatWell ${ }^{\circledR} 22 \%$-Crea Nutrition; ${ }^{\mathrm{b}}$ Analysis by Grain Growers Limited (NSW-Australia)/Acc. No. 66; ' Analysis with beta-glucan-glucan KIT from Megazyme (K-YBGL 04/2008) (Victoria, Australia); ${ }^{\mathrm{d}}$ Information based on nutrient fact sheet from Shiitake Mushroom Shenzhen Dashan Foodstuff Co. Ltd. NA: Not available.

recommended ranges of $10 \%$ to $100 \%$. At a particular speed, the viscosity was measured over the duration of 10 minutes. For the Shiitake mushroom beta-glucan, the minimum and maximum speeds used were $35 \mathrm{rpm}$ (shear rate of $263 \mathrm{~s}^{-1}$ ) and $55 \mathrm{rpm}$ (shear rate of $413 \mathrm{~s}^{-1}$ ), respectively, with an increment of $5 \mathrm{rpm}$. However, for the concentration $2 \mathrm{mg} / \mathrm{ml}$, the maximum speed possible before the torque exceeded $100 \%$ was $45 \mathrm{rpm}$. For the oat beta-glucan, since there was a significant difference in viscosity with the change of concentration, the range of speed that could be used (ensuring torque was between $10 \%-100 \%$ ) also differed considerably. For $3 \mathrm{mg} / \mathrm{ml}, 8$ $\mathrm{mg} / \mathrm{ml}$, and $2.5 \mathrm{mg} / \mathrm{ml}$, the speeds used varied from 20 to $55 \mathrm{rpm}, 8$ to $45 \mathrm{rpm}$, and 0.01 to $0.5 \mathrm{rpm}$, respectively.

\subsubsection{Determination of Molecular Weight}

Molecular weights of the oat and mushroom beta-glucans were determined by high performance liquid chromatography (LC-20AT, Shimadzu, Kyoto, Japan) using a refractive index detector (RID-10A, Shimadzu, Kyoto, Japan). Samples of $100 \mu \mathrm{L}$ were injected into a Waters Ultrahydrogel Linear $7.8 \times 300 \mathrm{~mm}$ size exclusion column (Waters, Milford, MA, USA) that was maintained at $50^{\circ} \mathrm{C}$ during measurement. De-ionised water (filtered 
Table 2. Composition of experimental diet.

\begin{tabular}{|c|c|c|c|c|c|c|c|}
\hline \multirow{2}{*}{ Composition of Experimental Diet } & \multicolumn{7}{|c|}{ Group } \\
\hline & LDO & MDO & HDO & LDM & MDM & HDM & HFD \\
\hline Carbohydrate (\% from total energy) & 33 & 33 & 33 & 33 & 33 & 34 & 32 \\
\hline Protein (\% from total energy) & 16 & 16 & 16 & 16 & 16 & 16 & 16 \\
\hline Fat (\% from total energy) & 49 & 49 & 48 & 49 & 49 & 48 & 50 \\
\hline Fibre (\% from total energy) & 2 & 2 & 3 & 2 & 2 & 3 & 2 \\
\hline \multicolumn{8}{|l|}{ Additional } \\
\hline Oat\% (wt:wt) & 0.9 & 2.7 & 8.1 & - & - & - & - \\
\hline Mushroom\% (wt:wt) & - & - & - & 0.7 & 2.1 & 6 & - \\
\hline Beta-glucan\% (wt:wt) & 0.2 & 0.6 & 1.8 & 0.2 & 0.6 & 1.8 & - \\
\hline Daily beta-glucan dose (g) & $0.2 \pm 0$ & $0.7 \pm 0.1$ & $1.9 \pm 0.3$ & $0.2 \pm 0$ & $0.5 \pm 0$ & $1.4 \pm 0.1$ & - \\
\hline Energy density (kJ/g) & 18.7 & 18.6 & 18.0 & 18.8 & 18.8 & 18.4 & 18.8 \\
\hline
\end{tabular}

The food was produced from a semi-synthetic material according to the recommendation of “AIN93 diets for laboratory rodents” [30].

Table 3. Viscosities of oat and shiitake mushroom solutions at different concentrations. The values presented here were obtained at $45 \mathrm{rpm}$, except for high dose oat which was obtained at $0.01 \mathrm{rpm}$.

\begin{tabular}{ccccc}
\hline & \multicolumn{2}{c}{ Oat } & \multicolumn{2}{c}{ Shiitake Mushroom } \\
\cline { 2 - 5 } Dose & $\begin{array}{c}\text { Concentration } \\
(\mathrm{mg} / \mathrm{ml})\end{array}$ & $\begin{array}{c}\text { Viscosity } \\
\text { (centipoises) }\end{array}$ & $\begin{array}{c}\text { Concentration } \\
(\mathrm{mg} / \mathrm{ml})\end{array}$ & $\begin{array}{c}\text { Viscosity } \\
\text { (centipoises) }\end{array}$ \\
\hline Low & 3 & $1.623 \pm 0.004$ & 2 & $0.812 \pm 0.003$ \\
Medium & 8 & $6.425 \pm 0.018$ & 6 & $1.056 \pm 0.019$ \\
High & 25 & $5089 \pm 6.932$ & 18 & $1.332 \pm 0.008$ \\
\hline
\end{tabular}

through a $0.45 \mu \mathrm{m}$ filter membrane) was the mobile phase, set to flow at $0.7 \mathrm{~mL} / \mathrm{min}$. The run time per sample was 30 minutes. Polyethylene glycol (PEG) with different molecular weights $(1000,3000,4000,6000$, and 8000 dalton) and pure beta-D glucan from Barley (Lot\# BCBC4494V, Sigma Aldrich, Sydney, Australia) with a molecular weight of 230,000 dalton, were used as standards. The concentrations of the standards were $15 \mathrm{mg} / \mathrm{ml}$. Oat and mushroom beta-glucan extracts, with concentrations $3 \mathrm{mg} / \mathrm{ml}$ and $2 \mathrm{mg} / \mathrm{ml}$ respectively (concentration in Table 3), were run through the HPLC together with the standards.

\subsection{Faecal Weight and Fat Content}

In the last week of intervention, faecal samples were collected each day for 3 days from all groups. The faecal samples from each cage ( 2 rats per cage) were collected, weighed and frozen at $-80^{\circ} \mathrm{C}$ until analysed for fat content. Fat content was analysed according to the modified procedure described by [31]. In brief, faeces were dried in a freeze dryer for $48 \mathrm{~h}$. The dried faecal samples were ground using a mortar and pestle. Fat from the ground dried faeces was extracted by stirring in chloroform- methanol 2:1, which was mixed well. The supernatant was recovered after centrifugation at $3000 \mathrm{rpm}$ for 5 minute at $22^{\circ} \mathrm{C}$. The supernatant was then dried using a stream of nitrogen. The dried fat was weighed using analytical balance (AND/HM-300, Japan). The ratio of faecal fat to faecal weight was calculated as $\mathrm{mg}$ faecal fat per $\mathrm{g}$ faecal weight.

\subsection{Statistical Analysis}

Data were expressed as mean \pm standard error of the mean (SEM). Data were analysis by two way ANOVA. Dunnet's method was used to examine the significance between the control group (HFD) and the treatment groups. To examine the significance among treatment groups, contrasts method was used followed by a post hoc-LSD test. A $p$ value of 0.05 or less was considered significant. Statistical analysis was performed using SAS 9.1 (SAS Institute, Cary, NC, USA) and SPSS software (version 17.0, SPSS Inc., Chicago, IL, USA).

\section{Results}

\subsection{Food Intake, Body Weight Gain and Fat Mass}

This study compared the dose response effects of oat and mushroom enrichment diets on the amount of food intake, body weight gain (BWG) and fat deposition of rats fed a high-fat diet (HFD). Food intake did not differ among the seven groups of dietary intervention (Table 4). The BWG was significantly affected by both diets and doses (Table 4). The rats fed with the mushroom enrichment diet had lower BWG compared with the oat enrichment diet (Figure $1 ; p=0.003$ ). Furthermore, when comparing between oats and mushroom powder there was no effect in the low dose $(p=0.743)$, but significant effect in the me- 


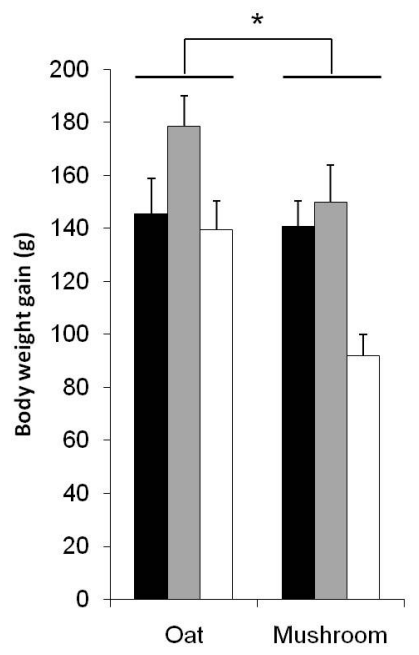

Figure 1. Body weight gain of male Wistar rats after 6 week consumption of HFD enriched with oat and mushroom. Data expressed as mean $\left( \pm\right.$ SEM). $\left(^{*}\right)$ significant $(p=0.003)$. $\square$ : LD; $\square$ : MD; $\square$ : HD.

dium dose $(-16 \%, p=0.040)$ and in the high dose $(-34 \%$, $p=0.002$ ) groups (Figure 1). The total fat deposition (epididymal + perirenal + omental + inguinal) was affected by both diets and doses (Table 5). The rats fed a mushroom enrichment diet had significantly lower total fat mass than the rats fed an oat enrichment diet (Figure 2(a), $p=0.001)$. Furthermore, there was no effect in total fat mass when comparing the low dose $(p=0.515)$ and medium dose ( $p=0.117$ ) groups, but significant effects were seen in the high dose $(-41 \%, p=0.015)$ group.

The WAT was affected by the diets and doses (Table 5). The rats fed a mushroom enrichment diet had significantly lower WAT than the rats fed an oat enrichment diet (Figure 2(b), $p=0.018$ ). Furthermore, there were no significant effects in WAT when comparing the low dose and medium dose groups but a trend towards an effect in the high dose $(-32 \%, p=0.072)$ group. The inguinal fat mass was affected by the diets and doses (Table 5). The rats fed a mushroom enrichment diet had significantly lower inguinal fat mass than the rats fed an oat enrichment diet (Figure 2(c), $p=0.006$ ). Furthermore, there were trends towards effects in the low dose $(-30 \%, p=$ $0.082)$, medium dose $(-32 \%, p=0.086)$ and high dose $(-47 \%, p=0.078)$ groups. No statistical differences were found in the amount of other fat mass (epididymal, perirenal, omental, and visceral) deposition between rats fed a mushroom and rats fed an oat enrichment diet.

\subsection{Plasma Triacylglycerol, Total Cholesterol, High Density Lipoprotein, and Non Esterified Fatty Acids}

Plasma TAG levels were significantly affected by the diets and doses (Table 6). The rats fed a mushroom en-

Table 4. Food intake and body weight gain in rats fed experimental diets.

\begin{tabular}{|c|c|c|c|c|c|c|c|c|}
\hline \multirow{2}{*}{ Parameter } & \multicolumn{3}{|c|}{ Oat group } & \multicolumn{3}{|c|}{ Mushroom group } & \multirow{2}{*}{ HFD } & \multirow{2}{*}{ ANOVA } \\
\hline & LD-O & MD-O & HD-O & LD-M & MD-M & HD-M & & \\
\hline Food Intake (g/day) & $24 \pm 1$ & $26 \pm 1$ & $24 \pm 1$ & $24 \pm 1$ & $24 \pm 1$ & $23 \pm 1$ & $24 \pm 1$ & \\
\hline Body weight gain (g) & $146 \pm 13$ & $179 \pm 11^{\#}$ & $139 \pm 11$ & $141 \pm 9$ & $150 \pm 14$ & $92 \pm 8$ & $129 \pm 8$ & Dt; Ds \\
\hline
\end{tabular}

Values are mean $( \pm$ SEM). Statistical analysis demonstrated \# $p<0.05$ compared to HFD. ANOVA, analysis of variance (two way); Dt, significant influence of diet $(p<0.05)$; Ds, Significant influence of doses $(p<0.05)$; HFD, high fat diet; LD-O, low dose oat; MD-O, medium dose oat; HD-O, high dose oat: LD-M, low dose mushroom; MD-M, medium dose mushroom; HD-M, high dose mushroom.

Table 5. Fat pad masses in rats fed experimental diets.

\begin{tabular}{|c|c|c|c|c|c|c|c|c|}
\hline \multirow{2}{*}{ Parameter } & \multicolumn{3}{|c|}{ Oat group } & \multicolumn{3}{|c|}{ Mushroom group } & \multirow{2}{*}{ HFD } & \multirow{2}{*}{ ANOVA } \\
\hline & LD-O & MD-O & HD-O & LD-M & MD-M & HD-M & & \\
\hline Epididymal & $13 \pm 1$ & $14 \pm 2$ & $12 \pm 1$ & $12 \pm 1$ & $13 \pm 1$ & $8 \pm 1$ & $11 \pm 1$ & $\mathrm{Dt}$ \\
\hline Perirenal & $16 \pm 2$ & $15 \pm 2$ & $13 \pm 2$ & $14 \pm 2$ & $13 \pm 2$ & $9 \pm 1^{\#}$ & $14 \pm 1$ & Dt $\times$ Ds \\
\hline Omental & $9 \pm 1$ & $11 \pm 1$ & $11 \pm 2$ & $11 \pm 1$ & $11 \pm 2$ & $7 \pm 1$ & $8 \pm 1$ & Dt $\times$ Ds \\
\hline Inguinal fat & $9 \pm 2$ & $10 \pm 2$ & $7 \pm 2$ & $7 \pm 1$ & $7 \pm 2$ & $4 \pm 0$ & $8 \pm 2$ & Dt:Ds \\
\hline Visceral fat $(\mathrm{E}+\mathrm{P}+\mathrm{O})$ & $38 \pm 4$ & $40 \pm 5$ & $34 \pm 4$ & $37 \pm 4$ & $34 \pm 4$ & $23 \pm 2$ & $34 \pm 2$ & Dt, Ds \\
\hline White adipose tissue (WAT) & $49 \pm 5$ & $51 \pm 7$ & $41 \pm 5$ & $44 \pm 4$ & $41 \pm 4$ & $28 \pm 2$ & $42 \pm 3$ & Dt, Ds \\
\hline Total fat masses & $49 \pm 5$ & $52 \pm 7$ & $41 \pm 5$ & $44 \pm 4$ & $41 \pm 4$ & $28 \pm 2$ & $43 \pm 3$ & Dt, Ds \\
\hline
\end{tabular}

ANOVA, analysis of variance (two way): Dt, significant influence of diet $(p<0.05)$; Ds, Significant influence of doses $(p<0.05)$; Dt $\times$ Ds, significant interaction between doses and diet $(p<0.05)$; HFD, High fat diet; LD-O, low dose oat; MD-O, medium dose oat; HD-O, high dose oat; LD-M, low dose mushroom; MD-M, medium dose mushroom; HD-M, high dose mushroom. 


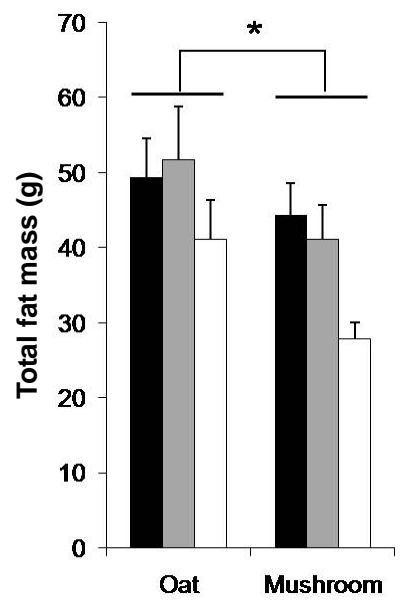

(a)

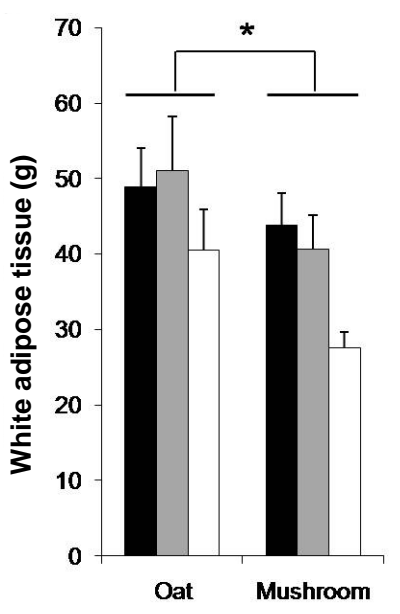

(b)

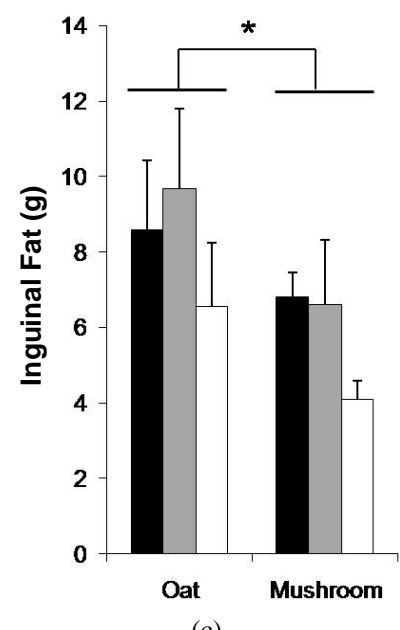

(c)

Figure 2. The total fat (a); White adipose tissue (WAT) (b) and Inguinal fat (c) after 6 week's consumption of HFD enriched with oat and mushroom. Data expressed as means $\left( \pm\right.$ SEM). $\left(^{*}\right)$ significant (a) $(p=0.001)$; (b) $(p=0.017)$; (c) $(p$ = 0.006). $\square$ : LD; $\square$ : MD; $\square$ : HD.

richment diet had significantly lower plasma TAG levels than the rats fed an oat enrichment diet (Figure 3, $p=$ 0.004). Furthermore, there were significant effects on plasma TAG in rats fed a mushroom enrichment diet in low $(-25 \%, p=0.022)$, medium $(-23 \%, p=0.035)$, and high dose $(-27 \%, p=0.011)$ groups compared to rats on oat enrichment diet. Plasma high density lipoprotein (HDL) levels were significantly affected by diet (Table 6). The rats fed a mushroom enrichment diet had significantly higher plasma HDL levels than the rats fed an oat enrichment diet $(p=0.002)$. There was a trend towards higher HDL levels in high doses of mushroom enrichment diet compared to the oat enrichment diet $(+22 \%, p$ $=0.052)$. There were no significant effects of diets and doses on plasma TC and non esterified fatty acids (NEFA) (Table 6).

\subsection{Oat and Mushroom Beta-Glucan Viscosity and Molecular Weight}

In order to find the mechanisms for the different effects of the oat and mushroom enrichment diets, this study characterized the beta-glucan viscosity and molecular

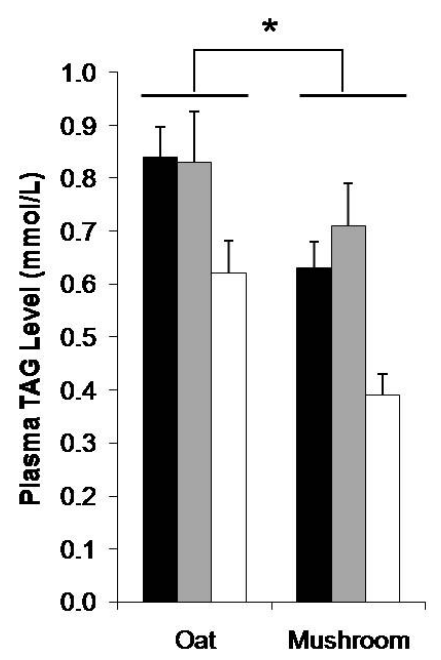

Figure 3. The plasma TAG level after 6 weeks consumption of HFD enriched with oat and mushroom. Data expressed as means $\left( \pm\right.$ SEM). $\left(^{*}\right)$ significant $(p=0.004)$. $\square$ : LD; $\square$ : MD; $\square$ : HD.

Table 6. Plasma lipid in rats fed experimental diets.

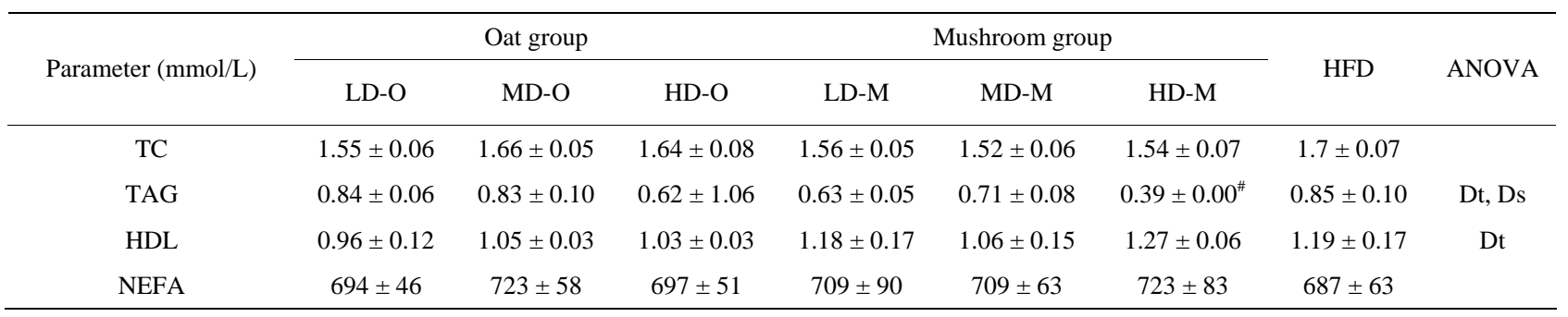

Values are mean $( \pm \mathrm{SEM})$. Statistical analysis demonstrated \# $p<0.05$ compared to HFD. ANOVA, analysis of variance (two way): Dt, significant influence of diet $(p<0.05)$; Ds, Significant influence of doses $(p<0.05)$; TC, total cholesterol; TAG, Triacylglycerol; HDL, high density lipoprotein; NEFA, non esterified fatty acid; HFD, high fat diet; LD-O, low dose oat; MD-O, medium dose oat; HD-O, high dose oat; LD-M, low dose mushroom; MD-M, medium dose mushroom; HD-M, high dose mushroom. 
weight from the oats and mushrooms. Generally, both the oat and shiitake mushroom beta-glucans showed sheer thinning behavior where the viscosity decreased with higher speed. At low speed, they also showed thixotropic behavior where the viscosity decreased over time under constant shear rate. Due to the variety of speeds used (to ensure that the torque exerted on the liquid was between the manufacturer's recommended range), for comparison, the viscosity data at $45 \mathrm{rpm}$ is presented (Table 3). At this speed, the viscosity of the oat beta-glucan, at the concentrations studied in this work, was considerably higher than that of the mushroom. The viscosity of mushroom beta-glucan even at the highest concentration (18 $\mathrm{mg} / \mathrm{ml}$ ) was lower than that of oat beta-glucan at the lowest concentration ( $3 \mathrm{mg} / \mathrm{ml})$, suggesting that viscosity is not the reason that mushroom beta-glucan is more effective to prevent weight gain and fat accumulation than oat beta-glucan. The viscosity data of high dose beta-glucan in oat solution $(25 \mathrm{mg} / \mathrm{ml})$ is shown separately because its value is 1000-fold higher (Table 3). Size exclusion chromatography data showed that both the Shiitake mushroom and oat extracts contained high molecular weight polymers, with those from Shiitake mushrooms larger than those from oats (Figure 4).

\subsection{The Weight of Faeces and Total Lipid in Faeces}

The faecal weights were significantly affected by the diets and doses (Table 7). The only diet that was statistically different to HFD was HD-O group showing a $24 \%$ reduction in faecal weight (Table 7).

The faecal fat weight to faecal weight ratio was significantly affected by doses in rats fed either mushroom or oat enrichment diets (Table 7). The faecal fat weight to faecal weight ratio was $16 \%$ higher in HD-M enrichment diet compared to HD-O ( $p=0.026)$. However, no differences in faecal fat weight to faecal weight ratio

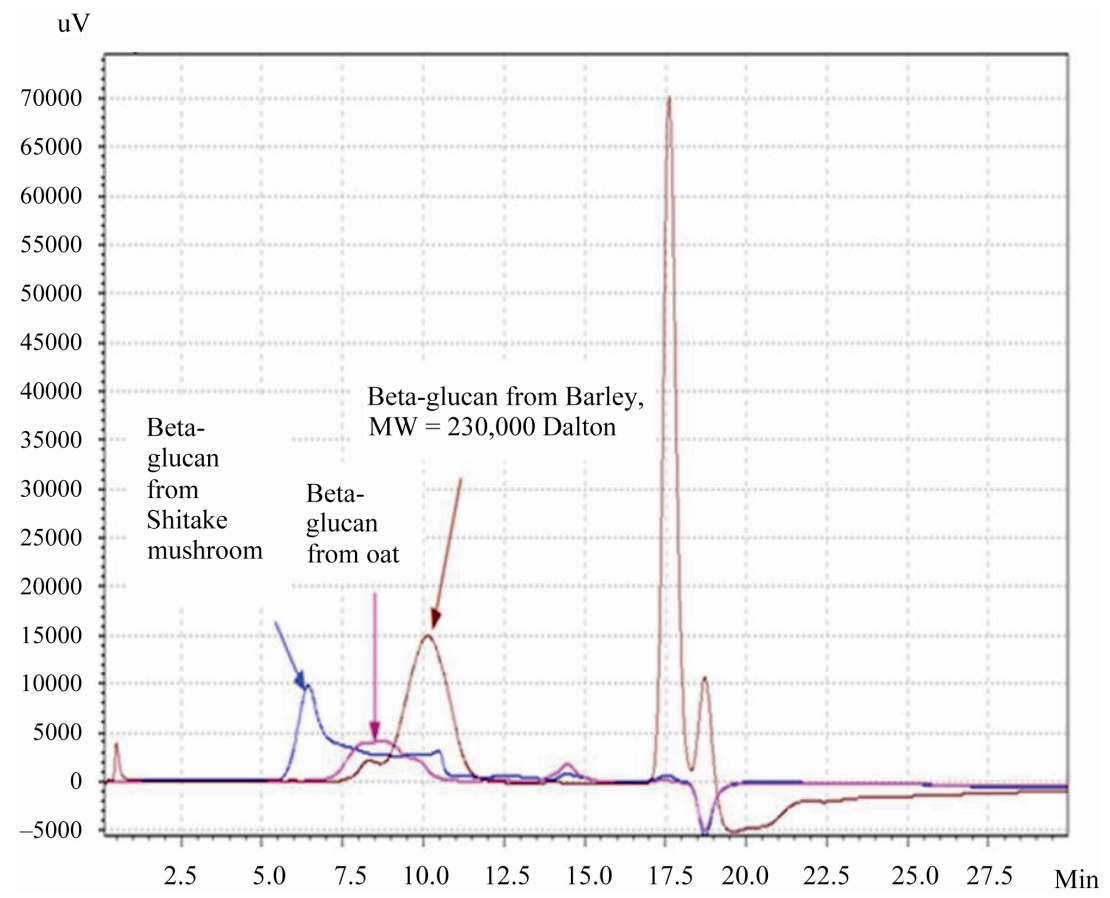

Figure 4. The high performance liquid chromatography (HPLC) profiles of $\beta$-glucan solutions extracted from oat and shitake mushroom and pure $\beta$ D-glucan from Barley.

Table 7. Plasma lipid in rats fed experimental diets.

\begin{tabular}{ccccccccrcr}
\hline \multirow{2}{*}{ Parameter } & \multicolumn{3}{c}{ Oat group } & \multicolumn{3}{c}{ Mushroom group } & \multirow{2}{*}{ HFD } & ANOVA \\
\cline { 2 - 7 } & LD-O & MD-O & HD-O & LD-M & MD-M & HD-M & & Dt; Ds \\
Faecal weight (g)/24 h & $3.9 \pm 0$ & $3.5 \pm 0.0$ & $3.1 \pm 0.2^{\#}$ & $3.9 \pm 0.1^{\#}$ & $3.7 \pm 0.2$ & $3.9 \pm 0.3$ & $4.1 \pm 0$ & \\
Faecal fat/24 h faecal weight & $12 \pm 0$ & $13 \pm 0$ & $16 \pm 0^{\#}$ & $12 \pm 0$ & $12 \pm 0$ & $19 \pm 0^{\#}$ & $12 \pm 0$ & Ds; Dt $\times$ Ds \\
\hline
\end{tabular}

Values are mean $\left( \pm\right.$ SEM). Statistical analysis demonstrated ${ }^{\#} p<0.05$ compared to HFD. ANOVA, analysis of variance (two way): Dt, significant influence of diet $(p<0.05)$; Ds, Significant influence of doses $(p<0.05)$; Dt $\times$ Ds, significant interaction between doses and diet $(p<0.05)$; HFD, high fat diet; LD-O, low dose oat; MD-O, medium dose oat; HD-O, high dose oat; LD-M, low dose mushroom; MD-M, medium dose mushroom; HD-M, high dose mushroom. 
were found between rats fed the same amount of low and medium doses of mushroom and oat enrichment diets.

\section{Discussion}

The present study for the first time found that rats fed a HFD enriched with shiitake mushroom powder had stronger effects in lowering body weight gain, fat deposition and plasma TAG, and compared with oat powder. To determine the beneficial effect of shiitake mushrooms, dosage must also be considered. This study showed that a high dose of mushroom enriched diet led to more exclusion of fat in the faeces compared to a high dose of oat enriched diet.

In general, animal studies have been previously reported in the literature and these showed that mushroom enriched diet had an effect on body weight gain prevention [24,32-34]. The data of BWG prevention by mushroom was depending on the rat species $[22,32,34,35]$, dosages in the diet and the variety of mushroom [32,33, 35-38]. There are no reported clinical trials in mushroom enriched diet to prevent BWG in humans.

The clinical study of oat enriched diet to prevent body weight gain was not largely reported, even though oat enrichment diet has been studied in different studies with different initial plasma lipid levels [5,7,11-14], doses of additional oat $[8,11,12]$, length of treatment $[5,7,12,13]$ and food matrix [5,7] all of which cannot achieve the constructive effect to prevent body weight gain. However a clinical study in oat enrichment diet reported decrease BWG when oat gives concurrently with exercise and low fat diet for 8 weeks [6]. Furthermore enriched oat in animal model diet showed decreasing in body weight gain when $20 \%$ oat bran concentrate enriched diet have $43 \%$ beta glucan content [10]. However, enrichment $4 \%$ and $8 \%$ oat beta glucan for replacing cellulose on hamster fed hypercholesterolemic diet did not affect on BWG [39].

There are very few studies that investigated the effect of oats and mushroom powder as part of a HFD on fat deposition. This current study showed that mushroom powder (HD-M) was more effective than oat powder (HDO) in lowering total fat mass (35\% vs 5\%), WAT (33\% vs $2 \%$ ) and inguinal fat ( $50 \%$ vs $13 \%$ ). Similar results were reported by [34] an additional 20\% of Maitake mushrooms in rats fed a cholesterol diet decreased $43 \%$ fat deposition. On the other hand no studies have reported the effect of oat enrichment diet on fat deposition.

It has been reported in the literature that mushroom and oat enriched diets have the effect of lowering plasma TAG in humans and animals. For example, mushroom enriched diets significantly lowered plasma TAG level in animal studies [22,23,33-35,40] and a human study [15], evidence from oat enriched diets showed that more than $20 \%$ of oats in the diet is necessary to achieve TAG lowering effects in rats [9]. Another study reported, the effect of an additional $20 \%$ oats with different molecular weight $(370.000 \mathrm{~g} / \mathrm{mol}-1450.000 \mathrm{~g} / \mathrm{mol})$ in mice fed HFD had a significant effect on lowering plasma TAG level [10]. However, in human studies, additional oats in the diet had no effect on plasma TAG lowering effect [58,11-13,17].

It is well known that lowering plasma TAG will decrease the fatty acid (FA) circulation, so it will decrease the source of fatty acid in adipose tissue through the adipogenesis process [41]. This was also reported by Novelli [4] that the increasing of plasma TAG level in rats fed HFD promoted fat deposition and BWG.

This current study showed that HD-M and HD-O resulted in $58 \%$ and $33 \%$, respectively, increase in faecal fat excretion per faecal weight compared to HFD. Studies have found that $40 \%$ oat enriched diets significantly increased faecal cholesterol fourfold increase in mice fed a high fat diet accompanied by a decreased plasma TC and TAG levels [9]. A clinical study also reported that an oat bran diet in healthy subjects increased fat in faeces 57\% more than a wheat bran diet [42]. Again, Cheung [36] reported hamsters fed HFD and Straw mushrooms enrichment significantly increased faecal sterol $81 \%$ more than hamsters fed the control diet (HFD and cellulose) which was accompanied by a significant $36 \%$ TC lowering effect.

Some studies reported the improvement in dyslipidaemia by beta-glucan was due to viscosity $[7,16,17]$. Both beta-glucan from oats [43,44] and mushrooms [45] can slow down the digestion transit time in the lower part of the small intestine and in the large intestine, therefore delaying nutrient absorption. This study measured both oat and mushroom beta-glucan molecular weight before these were added and mixed to the rat food. The results showed that MW in Shiitake mushroom beta glucan was higher than oat beta glucan used in this study. However, it is uncertain whether the viscosity of oats or mushrooms alone would be similar to the actual viscosity of rat food after it was added to oats or Shiitake mushrooms. This is because in food enriched with oats and Shiitake mushrooms viscosity was affected by the food preparation, such as food stored in a frozen state, and the digestion process that could decrease the MW of beta-glucan.

For instance, the freeze-thaw process will decrease MW by $40 \%$ and the digestion process will decrease by 100,000 Dalton [46]. In this study, the rat foods were frozen before being given to rats. As the mushroom MW was greater than oat MW, after the freeze-thaw process and digestion, the MW of the oats was expected to remain $<1,000,000$ Dalton and the MW of the mushrooms 
was expected to remain $>1,000,000$ Dalton. Although the correlation of MW and the effect of viscosity were still doubtful, Tosh, Brummer et al. [47] reported that in order to achieve the viscosity effect of beta-glucan, the MW should be $>1,000,000$ Dalton. Kerckhoffs, Hornstra et al. [7] also reported that a MW of beta-glucan $<1,200$,000 Dalton could still lower plasma lipid if administered with a drink. Therefore the viscosity in oats and Shiitake mushrooms tested before they were added to the rat food may not be able to explain the different effects of oat and Shiitake mushroom enriched diets in lowering plasma lipid levels in vivo.

Shiitake mushroom powder contains other active ingredients such as eritadenine and chitin. Eritadenine may contribute to the effect by inhibiting the plasma TAG derived lipoprotein release from the liver $[48,49]$. The concentration of eritadenine is about $0.36 \%$ in Shiitake mushroom powder [29]. The HD-M diet used in this study contains $\sim 200 \mathrm{mg}$ eritadenine/kg food. Sugiyama [18] reported that an additional $50 \mathrm{mg}$ eritadenine in choline deficient rats lowered plasma lipid by $-68 \%$ and $-83 \%$ of plasma TC and TAG respectively.

Chitin is insoluble in water and grouped as functional fibre to lower blood lipids [50,51]. However, chitin needs to be converted to chitosan to lower plasma lipid levels [52]. The process of converting chitin to chitosan is by alkaline hydrolysis $\left(45 \% \mathrm{NaOH}, 100^{\circ}\right)$ [53]. Hence this conversion is unlikely to have occurred in vivo, and therefore chitin is unlikely to explain the reduction in plasma TAG in this study.

Accordingly, this result and references suggest the lowering of plasma TAG levels in HD-M has possibly also been affected by eritadenine. This reason was supported by data that showed that although the fat faecal exclusion both of HD-M and HD-O were not significantly different the plasma TAG level, fat mass deposition and body weight gain between HD-O and HD-M were significantly different. Therefore other mechanisms possibly promoted the lowering of plasma TAG levels in HD-M.

\section{Conclusion}

In summary, the mushroom diet is better than the oat diet as part of a HFD because it reduces BWG, total fat mass, plasma TAG and increased fat faecal excretion. The dosage required for these effects was the HD-M (1.8\% wt:wt beta glucan from mushroom or $6 \%$ mushroom powder) with lower doses having minimal effects. The potential reason why mushrooms were better than oats was possibly its chemical structure. Food enriched with Shiitake mushroom was more stable and retained viscosity effects after the freeze-thaw process compared to food enriched with oats. In addition, Shiitake mushrooms also contain another potential biological component, namely eritadenine.

\section{Acknowledgements}

This study was supported by the School of Health Science, University of Wollongong (HDR student funding), IHMRI and URC grant of the University of Wollongong. Dian Handayani was supported by a DIKTI Post-graduate Scholarship for lecturers in Indonesian universities

\section{REFERENCES}

[1] G. Hotamisligil, "Inflammation and Metabolic Disorders," Nature, Vol. 444, No. 14, 2006, pp. 860-867. doi:10.1038/nature05485

[2] X.-F. Huang and J.-Z. Chen, “Obesity ComorbiditiesObesity, the PI3K/Akt Signal Pathway and Colon Cancer," Obesity Reviews, Vol. 10, No. 6, 2009, pp. 610-616. doi:10.1111/j.1467-789X.2009.00607.X

[3] J. Chen, A. Katsifis, C. Hu and X. Huang, "Insulin Decreases Therapeutic Efficacy in Colon Cancer Cell Line HT29 via the Activation of the PI3K/Akt Pathway,” Current Drug Discovery Technologies, Vol. 8, No. 2, 2011, pp. 119-125. doi:10.2174/157016311795563820

[4] E. Novelli, et al., "Anthropometrical Parameters and Markers of Obesity in Rats,” Laboratory Animals, Vol. 41, No. 1, 2007, pp. 111-119.

[5] K. Behall, D. Scholfield and J. Hallfrisch, "Effect of Beta-Glucan Level in Oat Fibre Extracts on Blood Lipids in Men and Women," Journal of the American College of Nutrition, Vol. 16, No. 1, 1997, pp. 46-51.

[6] N. Reyna-Villasmil, et al., "Oat-Derived b-Glucan Significantly Improves HDLC and Diminishes LDLC and Non-HDL Cholesterol in Overweight Individuals with Mild Hypercholesterolemia," American Journal of Therapeutics, Vol. 14, 2007, pp. 203-212. doi:10.1097/01.pap.0000249917.96509.e7

[7] D. Kerckhoffs, G. Hornstra and R. Mensink, "Cholesterol-Lowering Effect of Beta-Glucan from Oat Bran in Mildly Hypercholesterolemic Subjects May Decrease When Beta-Glucan Is Incorporated into Bread and Cookies," American Journal of Clinical Nutrition, Vol. 78, No. 4, 2003, pp. 221-227.

[8] K. Maki, et al., "Whole-Grain Ready-to-Eat Oat Cereal, as Part of a Dietary Program for Weight Loss, Reduces Low-Density Lipoprotein Cholesterol in Adults with Overweight and Obesity More than a Dietary Program Including Low-Fiber Control Foods,” Journal of American Diet Association, Vol. 110, 2010, pp. 205-214.

[9] K. Andersson, K. Svedberg, M. Lindholmb, R. Ostec and P. Hellstranda, "Oats (Avena sativa) Reduce Atherogenesis in LDL-Receptor-Deficient Mice,” Atherosclerosis, Vol. 212, No. 1, 2010, pp. 93-99.

[10] I. Bae, S. Lee, S. Kim and H. Lee, "Effect of Partially Hydrolyzed Oat b-Glucan on the Weight Gain and Lipid Profile of Mice," Food Hydrocolloids, Vol. 23, No. 7, 
2009, pp. 2016-2021.

[11] W. Karmally, et al., "Cholesterol-Lowering Benefits of Oat-Containing Cereal in Hispanic Americans,” Journal of American Dietetic Association, Vol. 105, No. 6, 2005, pp. 967-970.

[12] J. Lovegrove, A. Clohessy, H. Milon and C. Williams, "Modest Doses of b-Glucan Do Not Reduce Concentrations of Potentially Atherogenic Lipoproteins 1 - 3," American Journal of Clinical Nutrition, Vol. 72, No. 1, 2000, pp. 49-55.

[13] K. Queenan, et al., "Concentrated Oat $\beta$-Glucan, a Fermentable Fiber, Lowers Serum Cholesterol in Hypercholesterolemic Adults in a Randomized Controlled Trial," Nutrition Journal, Vol. 6, No. 6, 2007, pp. 1-8.

[14] K. Maki, et al., "Lipid Responses to Consumption of a Beta-Glucan Containing Ready-to-Eat Cereal in Children and Adolescents with Mild-to-Moderate Primary Hypercholesterolemia," Nutrition Research, Vol. 23, 2003, pp. 1527-1535.

[15] I. Schneider, et al., "Lipid Lowering Effects of Oyster Mushroom (Pleurotus ostreatus) in Humans," Journal of Functional Foods, Vol. 3, 2011, pp. 17-27.

[16] M. U. Beer, E. Arrigoni and R. Amado, "Effects of Oat Gum on Blood Cholesterol Levels in Healthy Young Men,” European Journal of Clinical Nutrition, Vol. 49, No. 7, 1995, p. 517.

[17] A. Lia, et al., "Oat Beta-Giucan Increases Bile Acid Excretion and a Fiber-Rich Barley Fraction Increases Cholesterol Excretion in Ileostomy Subjects 1 - 3," American Journal of Clinical Nutrition, Vol. 62, 1995, pp. 12451251.

[18] K. Sugiyama, T. Akachi and A. Yamakawa, "EritadenineInduced Alteration of Hepatic Phospholipid Metabolism in Relation to Its Hypocholesterolemic Action in Rats," The Journal of Nutritional Biochemistry, Vol. 6, No. 2, 1995, pp. 80-87. doi:10.1016/0955-2863(94)00017-G

[19] K. Sugiyama, A. Yamakawa, H. Kawagishi and S. Saeki, "Dietary Eritadenine Modifies Plasma Phosphatidylcholine Molecular Species Profile in Rats Fed Different Types of Fat," The Journal of Nutrition, Vol. 127, No. 4, 1997, pp. 593-599.

[20] Y. Shimada, T. Morita and K. Sugiyama, "Eritadenineinduced Alterations of Plasma Lipoprotein Lipid Concentrations and Phosphatidylcholine Molecular Species Profile in Rats Fed Cholesterol-Free and Cholesterol-Enriched Diets," Bioscience, Biotechnology and Biochemistry, Vol. 67, No. 5, 2003, pp. 996-1006. doi:10.1271/bbb.67.996

[21] J. J. Volman, J. D. Ramakers and J. Plat, "Dietary Modulation of Immune Function by Beta-Glucans,” Physiology \& Behavior, Vol. 94, No. 2, 2008, pp. 276-284. doi:10.1016/j.physbeh.2007.11.045

[22] C. Xu, H. Y. Zhong, J. H. Zeng and G. Jing, “The Pharmacological Effect of Polysaccharides from Lentinus Edodes on the Oxidative Status and Expression of VCAM1mRNA of Thoracic Aorta Endothelial Cell in HighFat-Diet Rats," Carbohydrate Polymers, Vol. 74, No. 3, 2008, pp. 445-450. doi:10.1016/j.carbpol.2008.03.018
[23] K. Takashima, C. Sato, Y. Sasaki, T. Morita and S. Takeyama, "Efect of Ertadenine on Cholesterol Meatbolisme in the Rat,” Biochemical Pharmachology, Vol. 23, No. 2, 1974, pp. 433-438.

[24] A. M. Neyrinck, et al., "Dietary Supplementation with Chitosan Derived from Mushrooms Changes Adipocytokine Profile in Diet-Induced Obese Mice, a Phenomenon Linked to Its Lipid-Lowering Action,” International Immunopharmacology, Vol. 9, No. 6, 2009, pp. 767-773. doi:10.1016/j.intimp.2009.02.015

[25] L. Sjoblom and A. Eklund, "Determination of HDL2 Cholesterol by Precipitation with Dextran Sulfate and Magnesium Chloride: Establishing Optimal Conditions for Rat Plasma,” Lipids, Vol. 24, 1989, pp. 532-534.

[26] P. Mattila, et al., "Contents of Vitamins, Mineral Elements, and Some Phenolic Compounds in Cultivated Mushrooms," Journal of Agricultural and Food Chemistry, Vol. 49, No. 5, 2001, pp. 2343-2348.

[27] A. Beck, S. Tosh, M. Batterham, L. Tapsel and X. Huang, "Oat Beta-Glucan Increases Postprandial Cholecystokinin Levels, Decrease Insulin Response and Extends Subjective Satiety in Overweight Subjects," Molecular Nutrtion \& Food Research, Vol. 53, No. 10, 2009, pp. 1-9.

[28] C. Dikeman, L. Bauer, A. Flickinger and G. Fahey, "Effects of Stage of Maturity and Cooking on the Chemical Composition of Select Mushroom Varieties,” Journal of Agricultural and Food Chemistry, Vol. 53, No. 4, 2005, pp. 1130-1138.

[29] J. Enman, U. Rova and K. Berglund, "Quantification of the Bioactive Compound Eritadenine in Selected Strains of Shiitake Mushroom (Lentinus edodes)," Journal of Agricultural and Food Chemistry, Vol. 55, No. 4, 2007, pp. 1177-1180.

[30] P. G. Reeves, “Component of the AIN-93 Diets as Improvements in the AIN-76A Diet," The Journal of Nutrition, Vol. 127, No. 5, 1997, pp. 838S-841S.

[31] D. Kritchevsky and S. A. Tepper, "Influence of a Fiber Mixture on Serum and Liver Lipids and on Fecal Fat Excretion in Rats," Nutrition Research, Vol. 25, No. 5, 2005, pp. 485-489. doi:10.1016/j.nutres.2005.02.001

[32] J.-R. Noh, et al., “A Phellinus baumii Extract Reduces Obesity in High-Fat Diet-Fed Mice and Absorption of Triglyceride in Lipid-Loaded Mice,” Journal of Medicinal Food, Vol. 14, No. 3, 2011, pp. 209-218. doi:10.1089/jmf.2010.1152

[33] W.-W. Kim, K.-H. Kim, H.-J. Choi and D.-S. Lee, "AntiDiabetic Activity of b-Glucans and Their Enzymatically Hydrolyzed Oligosaccharides from Agaricus blazei," Biotechnology Letters, Vol. 27, No. 7, 2005, pp. 483-487.

[34] K. Kubo and H. Nanba, "The Effect of Maitake Mushroom on Liver and Serum Lipids," Alternative Therapies in Health and Medicine, Vol. 2, No. 5, 1996, pp. 62-66.

[35] N. Talpur, et al., "Antihypertensive and Metabolic Effects of Whole Maitake Mushroom Powder and Its Fractions in Two Rat Strains," Molecular and Cellular Biochemistry, Vol. 237, No. 1, 2002, pp. 129-136.

[36] P. Cheung, "Plasma and Hepatic Cholesterol Levels and 
Fecal Neutral Sterol Excretion Are Altered in Hamsters Fed Straw Mushroom Diets," The Journal of Nutrition, Vol. 128, No. 9, 1998, pp. 1512-1516.

[37] K. Mori, C. Kobayashi, T. Tomita, S. Inatomi and M. Ikeda, "Antiatherosclerotic Effect of the Edible Mushroom Pleurotus eryngii (Eringi), Grifola frondosa (Maitake), and Hypsizygus marmoreus (Bunashimeji) in Apolipoprotein E-Mice Deficiency Mice," Nutrition Research, Vol. 28, No. 5, 2008, pp. 335-342.

[38] M. Fukushima, T. Ohashi, Y. Fujiwara, K. Sonoyama and M. Nakano, "Cholesterol-Lowering Effects of Maitake (Grifola frondosa) Fiber, Shiitake (Lentinus edodes) Fiber, and Enokitake (Flammulina velutipes) Fiber in Rats," Society for Experimental Biology and Medicine, Vol. 226, No. 8, 2001, pp. 758-765.

[39] B. Delaney, et al., "Beta Glucan Fraction from Barley and Oats Are Similarly Antiatherogenic in Hypercholesterolemic Syrian Golden hamster," The Journal of Nutrition, Vol. 133, 2003, pp. 468-475.

[40] Y. Kabir and S. Kimura, "Dietary Mushrooms Reduce Blood Pressure in Spontaneously Hypertensive Rats (SHR),” Journal of Nutritional Science and Vitaminology (Tokyo), Vol. 35, No. 1, 1989, pp. 91-94. doi:10.3177/jnsv.35.91

[41] P. J. Voshol, P. C. N. Rensen, K. W. van Dijk, J. A. Romijn and L. M. Havekes, "Effect of Plasma Triglyceride Metabolism on Lipid Storage in Adipose Tissue: Studies Using Genetically Engineered Mouse Models,” Biochimica et Biophysica Acta (BBA)-Molecular and Cell Biology of Lipids, Vol. 1791, No. 6, 2009, pp. 479485.

[42] H.-L. Chen, V. Haack, C. Janecky, N. Vollendorf and J. Marlett, "Mechanisms by Which Wheat Bran and Oat Bran Increase Stool Weight in Humans," American Journal of Clinical Nutrition, Vol. 68, No. 1998, pp. 711-719.

[43] E. Lund, J. Gee, J. Brown, P. Wood and I. Johnson, "Effect of oat Gum on the Physical Properties of the Gastrointestinal Contents and on the Uptake of D-Galactose and Cholesterol by Rat Small Intestine in Vitro," British Journal of Nutrition, Vol. 62, 1989, pp. 91-101.

[44] F. Manthey, G. Hareland and D. Ghuseby, "Soluble and Insoluble Fibre Content and Composition in Oat," Cereal Chemistry, Vol. 76, No. 3, 1999, pp. 417-420. doi:10.1094/CCHEM.1999.76.3.417
[45] F. Guo, et al., "Effects of Mushroom and Herb Polysaccharides, as Alternatives for an Antibiotic, on the Cecal Microbial Ecosystem in Broiler Chickens," Poultry Science, Vol. 83, No. 2, 2004, pp. 175-182.

[46] Y. Brummer, P. Wood, S. Tosh, X. Lan-Pidhainy and T. Wolever, "Modification of Molecular Weight and Solubility of Beta Glucan in Oat Bran Muffins, and Effect on the Glycemic Response," Proceedings of AACC Meeting; World Grains Summit: Foods and Beverages, San Francisco, 2006.

[47] S. M. Tosh, Y. Brummer, T. M. S. Wolever and P. J. Wood, "Glycemic Response to Oat Bran Muffins Treated to Vary Molecular Weight of Beta-Glucan," Cereal Chemistry, Vol. 85, No. 2, 2008, pp. 211-217.

[48] Z. Yao and D. Vance, "The Active Synthesis of Phosphatidylcholine Is Required for Very Low Density Lipoprotein Secretion from Rat Hepatocy," The Journal of Biological Chemistry, Vol. 263, No. 6, 1988, pp. 29983004.

[49] R. Jacobs, C. Devlin, I. Tabas and D. Vance, "Targeted Deletion of Hepatic CTP: Phosphocholine Cytidylyltransferase in Mice Decreases Plasma High Density and Very Low Density Lipoproteins," The Journal of Biological Chemistry, Vol. 279, No. 45, 2004, pp. 47402-47410. doi:10.1074/jbc.M404027200

[50] J. Van Der Kamp, N.-G. Asp, J. Jones and G. Schaafsma, "Dietary Fibre, Bio-Active Carbohydrates for Food and Feed,” In: N.-G. Asp, Ed., Definition and Analysis of Dietary Fibre in the Context of Food Carbohydrate, Wageningen Academic Publisher, Wageningen, 2003, p. ill.

[51] N. Nwe and W. Stevens, "Chitosan Isolation from the Chitosan-Glucan Complex of Fungal Cell Wall Using Amylolytic Enzymes,” Biotechnology Letters, Vol. 24, No. 18, 2002, pp. 1461-1464.

[52] S. S. Koide, "Chitin-Chitosan: Properties, Benefits and Risks,” Nutrition Research, Vol. 18, No. 6, 1998, pp. 10911101. doi:10.1016/S0271-5317(98)00091-8

[53] L.-K. Han, Y. Kimura and H. Okuda, "Reduction in fat Storage during Chitin-Chitosan Teratment in Mice and High Fat Diet,” International Journal of Obesity, Vol. 23, 1999, pp. 174-179. 